\title{
Management of orthopaedic and traumatology patients during the Coronavirus disease (COVID-19) pandemic in northern Italy
}

\author{
Pietro Simone Randelli ${ }^{1,2,3} \cdot$ Riccardo Compagnoni $^{1,2,3}$
}

Received: 24 March 2020 / Accepted: 21 April 2020 / Published online: 25 April 2020

(c) European Society of Sports Traumatology, Knee Surgery, Arthroscopy (ESSKA) 2020

\begin{abstract}
Purpose This article aims to share northern Italy's experience in hospital re-organization and management of clinical pathways for traumatic and orthopaedic patients in the early stages of the COVID-19 pandemic.

Methods Authors collected regional recommendations to re-organize the healthcare system during the initial weeks of the COVID-19 pandemic in March, 2020. The specific protocols implemented in an orthopaedic hospital, selected as a regional hub for minor trauma, are analyzed and described in this article.

Results Two referral centres were identified as the hubs for minor trauma to reduce the risk of overload in general hospitals. These two centres have specific features: an emergency room, specialized orthopaedic surgeons for joint diseases and trauma surgeons on-call 24/7. Patients with trauma without the need for a multi-disciplinary approach or needing non-deferrable elective orthopaedic surgery were moved to these hospitals. Authors report the internal protocols of one of these centres. All elective surgery was stopped, outpatient clinics limited to emergencies and specific pathways, ward and operating theatre dedicated to COVID-19-positive patients were implemented. An oropharyngeal swab was performed in the emergency room for all patients needing to be admitted, and patients were moved to a specific ward with single rooms to wait for the results. Specific courses were organized to demonstrate the correct use of personal protection equipment (PPE).

Conclusion The structure of the orthopaedic hubs, and the internal protocols proposed, could help to improve the quality of assistance for patients with musculoskeletal disorders and reduce the risk of overload in general hospitals during the COVID-19 pandemic.
\end{abstract}

Keywords Orthopaedic $\cdot$ Coronavirus $\cdot$ SARS-CoV-2 $\cdot$ Traumatology $\cdot$ Triage $\cdot$ Pathways

\section{Introduction}

Coronavirus disease (COVID-19) is a severe acute respiratory syndrome determined by a new type of Coronavirus, which was declared a pandemic by the world health

Riccardo Compagnoni

riccardo.compagnoni@gmail.com

1 Laboratory of Applied Biomechanics, Department of Biomedical Sciences for Health, Università degli Studi di Milano, Via Mangiagalli 31, 20133 Milan, Italy

$21^{\circ}$ Clinica Ortopedica, ASST Centro Specialistico Ortopedico Traumatologico Gaetano Pini-CTO, Piazza Cardinal Ferrari 1, 20122 Milan, Italy

3 Research Center for Adult and Pediatric Rheumatic Diseases (RECAP-RD), Department of Biomedical Sciences for Health, Università degli Studi di Milano, Via Mangiagalli 31, 20133 Milan, Italy organization on March 11, 2020 [3]. The Coronavirus, COVID-19, is affecting 167 countries around the world, most notably in the USA, Italy, China, Iran and Spain [2, 4]. As of 20 March 2020, Italy was the world's centre of active Coronavirus cases and more than half of these cases were diagnosed in Lombardy, a region inhabited by 10 million people in the north of Italy, with 22,264 positive patients and 2,549 deaths in less than 4 weeks (21 February-20 March 2020), 1,050 patients are admitted into intensive care units and 8,785 patients in internal medicine wards on 20 March 2020 [1]. The regional healthcare system had to quickly change its organization to meet the needs of positive patients, considering their frequent hospitalizations ( 8,785 people, $39.4 \%$ of symptomatic patients) and admittance to intensive care units (ICU) (1,050 people, $4.7 \%$ of symptomatic patients) [1]. On 8 March 2020 the regional government decided to redirect patients affected by severe acute pathologies to selected centres that could cater to their 
specific requirements. These hospitals must guarantee a $24 \mathrm{~h}$ service with active medical rounds, with different pathways between COVID-19-positive and -negative patients and should cooperate with surrounding hospitals for patient management. The decision was to admit suspected COVID-19 patients in 15 first-responder hub hospitals, chosen because they either had expertise in infectious disease or were part of the Venous-Venous ECMO Respiratory Failure Network. To make ICU beds and qualified personnel available, nonurgent procedures were cancelled, and over the first 18 days, 482 ICU beds were made available for COVID-19 patients. Several specific hubs were selected for diseases other than COVID-19, like trauma, neurosurgical, neurologic, cardiological and vascular emergencies. Focusing on patients with orthopaedic and trauma emergencies, two different types of hubs were selected; the poly-specialistic big trauma centres and specialistic referral centres for minor trauma or elective orthopaedic surgeries that cannot be postponed.

We aim to share our experience of regional hospital reorganization and management of clinical pathways for traumatic and orthopaedic patients, during the early weeks of the COVID-19 pandemic.

\section{Selection of orthopaedic and trauma regional referral centres}

The rationale of regional healthcare decisions was to make available specialized human, technological and hospital resources for the treatment of patients with COVID-19 and severe acute respiratory syndrome [5]. This approach led to implementing the hub-and-spoke organizational design, defined as a model which arranges service delivery assets into a network, consisting of an anchor establishment (hub) which offers a full array of services, complemented by secondary establishments (spokes) which offer more limited service arrays, routing patients needing more intensive services to the hub for treatment. Lombardy has routinely six poly-trauma centres, covering about 1,600,000 people each. For the COVID-19 pandemic emergency, the regional decision was to reduce them to three poly-trauma hubs and to modulate the network accordingly.

Two referral centres were identified as the hubs for minor trauma or non-deferrable elective orthopaedic surgeries: "Gaetano Pini orthopedic institute, Milano" and "Galeazzi orthopedic institute, Milano". These two centres had specific features: an emergency room, specialized orthopaedic surgeons for joint diseases and trauma surgeons on-call 24/7. All minor trauma without the need for a multi-disciplinary approach and requiring surgical treatment was considered appropriate for moving into one of these two hospitals. Different considerations were made for non-postponable elective orthopaedic surgery. An expert meeting was organized among orthopaedic specialists in the referral centres to define a list including all the pathologies that could be considered as emergencies. This list included generic descriptions of the pathologies, not limited to specific joints, to permit a broad interpretation of the particular emergency in question. The list was delivered to all the hospitals of the Lombardy region, and the English translation of the final version is reported in Table 1. Patient transfer was possible after phone contact with the specialist of the hub centre, and after completing a checklist to evaluate clinical aspects such as fever, flu-like or respiratory symptoms, swabs and medical history.

\section{The specific algorithm adopted in one of the referring orthopaedics and traumatology centres}

The authors describe the internal protocol developed in one of the two hubs for minor trauma and non-deferrable orthopaedic elective surgery, Orthopedic institute Gaetano Pini, Milano. This orthopaedic centre is the oldest orthopaedic institute in Italy, founded in 1874, and its activity is focused on musculoskeletal diseases, covering all aspects of orthopaedics and trauma. The hospital has an emergency service open $24 \mathrm{~h}$ every day, and a trauma surgeon on-call for emergencies. The emergency service treats about 35,000 patients every year, with more than 2,000 hospitalizations. The hospital has 12 operating theatres working on weekdays, with about 7,400 surgical cases performed every year, of which about $30 \%$ are fractures. Public outpatient service is active all weekdays for first consultations, and post-operative controls, as well as a private outpatient clinic, are active all working days in the same building.

After the outbreak of the COVID-19 in the region, the first implementation of the hospital was the creation of an institutional "Crisis unit" to facilitate rapid and efficient

Table 1 List of pathologies considered elective surgery not deferrable

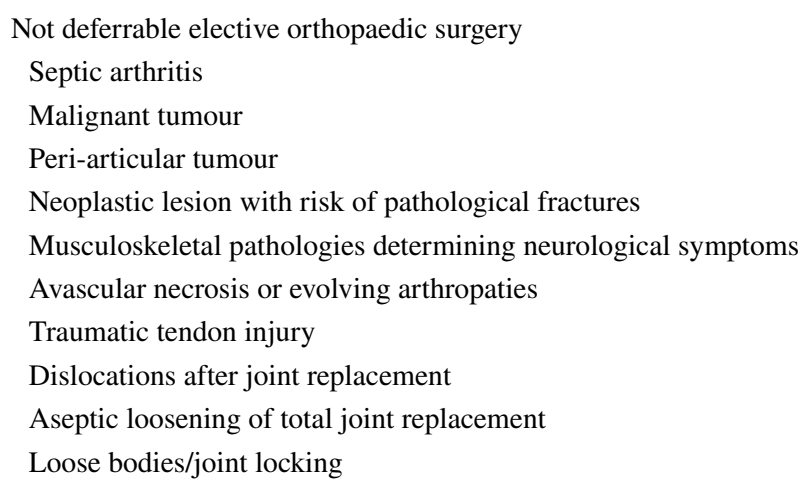

This list was shared with all regional hospital and patients are directed to one of the referral centres for minor trauma 
action. This team consisted of the general manager of the hospital, the chief of anaesthesiology, the chief of the orthopaedic department, the scientific director, hospital medical director, risk manager, head of the nursing team and the communication manager. A daily meeting was organized by the team to monitor the evolution of COVID-19, implement regional recommendations and take decisions regarding employers' organization, surgical and outpatient activities, emergency department procedures and management of suspicious Coronavirus infections among patients and workers.

The first decision was to rationalize surgical activity, blocking all elective surgery and performing only trauma cases. The rationale of this decision was to avoid a potential overlapping of a Coronavirus infection with the post-operative course so as not to risk a transfer to ICU. This aspect is relevant, considering that two potential risks underlie a transfer in ICU. The first is the potential lack of beds due to the large number of patients hospitalized for COVID-19, exposing the operated patient to a life-threatening risk. The second one is to leave available all potential emergency beds available during the outbreak of a disease that requires ICU in a high percentage of the infected.

Four operating rooms were organized for trauma cases or non-deferrable orthopaedic surgery. An operating theatre with specific pathways for nurses and surgeons was organized for the treatment of positive or potentially positive patients.

The second decision was to modulate outpatient clinics to reduce overcrowding in the waiting rooms. All public and private first consultations were stopped, excluding the priority ones identified by general practitioners with a specific emergency request. Every case of post-operative controls was evaluated and, if considered necessary, the agenda was designed to guarantee at least $15 \mathrm{~min}$ for consultation. Patients with neoplastic diseases, rheumatic diseases, plaster removal, Parkinson and neurological diseases were noted. Patients had to come to the consultation alone, and in cases of babies or dependent people, only one accompanying person was allowed.

The third decision was to regulate access to the hospital, both for employees and patients. Only one gateway was maintained, with body temperature screening and a station for hand washing with an alcoholic solution. Access was only permitted to people with a booked appointment or medical test. In visiting hours, access was only permitted to one relative for hospitalized patients and was not possible for COVID patients.

The fourth implementation was to optimize hospital human resources to reduce the need for physical presence in the structure. Considering the reduction of clinical activity, a medical staff shift and rest period were organized to guarantee efficient clinical activity but to minimize presence in the hospital. Administrative personnel activity was reduced and, when possible, remote working was implemented.

The fifth implementation was the conversion of a hospital ward to a COVID-19 exclusive patient ward. In this sector, it was planned to hospitalize patients with a positive COVID19 test, from the emergency department rather than from different wards. This area was restricted to selected trained employees, with the use of personal protection equipment (PPE) following World Health Organization and regional recommendations. An oropharyngeal swab was performed in the emergency room for all patients needing to be admitted, and patients were moved to a specific ward made up of single rooms to wait for results.

\section{Personal protection equipment (PPE)}

COVID-19 was thought to spread mainly from person-toperson, between people who were in close contact with one another (within about $1 \mathrm{~m}$ ) and through respiratory droplets produced when an infected person coughs or sneezes. Health-care workers in the hospital were trained to adopt the correct behaviours and wear the correct personal protection equipment when required. A significant lack of PPE in the whole Lombardy region was noted at the beginning of the COVID-19 outbreak. Measures were also taken to raise awareness among staff regarding correct PPE use to avoid the reduction of the available stock. Regional guidelines regarding the use of PPE were shared by the hospital educational office both with residential and online-learning courses implemented to be more effective. The residential courses had to be structured in large meeting rooms, with all the attendees using a protection surgical mask and with a limited number of participants. On March 18, the regional department for health shared the following recommendations [3].

General recommendations suggest on staying at least $1 \mathrm{~m}$ from other people, performing frequent hand wash and reducing the number of people in the hospital, employed and visitors, to minimize the risk of contamination.

Implementation of more PPE was recommended in some specific situations: execution of oropharyngeal swab, work in emergency triage and assistance of patients with respiratory illness.

During the execution of the oropharyngeal swab, it was suggested to use a mask that respects the FFP2 standard (filtering facepiece 2), a protective disposable gown, gloves, and protective glasses.

Healthcare workers working in emergency triage had to stay at least $1 \mathrm{~m}$ from the patients, wear surgical protection mask and use an intercom or a working station with a partition glass. In the case of a clear respiratory illness, the 
patient should be moved immediately into a single room with good air exchange and should wear a surgical mask.

\section{Surgical and anaesthesiology team: specific recommendations}

Medical doctors, nurses and support healthcare workers had to pay particular attention to respect the new dressing and undressing procedures and for patients access, staying and discharging in the operating room. The hospital created a specific ward and pathway for COVID-19-positive patients. As described before, this included the use of dedicated corridors, an elevator and an isolated operating room with limited access to personnel.

Anaesthetic procedures had to be performed with particular attention, due to the potential spread of infected droplets and aerosol. The period which represents the highest risk of exposure involved direct contact with respiratory droplets during airway management, primarily during intubation and extubation. For this reason, a specific protection kit was implemented in this procedure and all intubations were performed using a video laryngoscope to keep the head of the anaesthesiologist away from the patient's mouth and to allow a faster procedure. Furthermore, inadequate personal protective equipment (PPE), improper use of PPE and poor hand hygiene were potential factors that can lead to virus transmission. Where possible, local or spinal anesthesia was preferred in COVID-19 patients to reduce the risk of overlapping pneumonia secondary to an invasive procedure. The anaesthesiologist and the dedicated nurse should dress in a filter room with an FPP2 mask, a protective disposable gown that includes the neck and head, protective glasses and disposable protection for the shoes. Healthcare professionals not involved in the procedure had to wait outside the operating room to reduce the risk of contamination. The surgeon and surgical nurse dressed in the new isolated changing room. After a social (routine) hand wash, second-skin gloves were used, and not removed until the final PPE removal at the end of all the procedures. Surgical dressings included a protective disposable gown that included the neck and head, protective glasses and disposable protection for the shoes. PPE had to be applied correctly in the proper order before entry into the patient care area and should not be modified while in the patient care area. Removing used PPE is a high-risk process that required a structured procedure, a trained observer, an assistant and a designated area for removal to ensure protection. PPE had to be removed slowly in the correct sequence to reduce the possibility of self-contamination or other exposure to the virus.

\section{Management in the emergency department of patients with trauma diseases}

The emergency department was re-arranged to deal with the increased activity due to the status of a regional hub. The "crisis unit" created three different flowcharts for different types of patients:

- patients needing immediate surgical treatment;

- patients needing hospitalization for planning surgical treatment;

- patients to be treated conservatively.

\section{Patients needing immediate surgical treatment (Fig. 1)}

This group included patients referred to the emergency department with a severe, acute traumatic condition requiring immediate surgical treatment. The clinical pathway started with body temperature screening and evaluation of flu-like symptoms. An oropharyngeal swab was performed in all patients admitted, using the PPE previously described (FPP2 mask, protective disposable gown, gloves and protective glasses). Patients were then moved to the operating theatre and the surgical procedure was performed. At the end of the procedure, the patients were moved to the dedicated ward, waiting in a single room for the result of the oropharyngeal swab. In the case of a negative result, the patients were moved to the usual ward. In the case of a positive result, the patient was moved to the dedicated COVID-19 ward. This pathway included dedicated corridors, an elevator and an isolated operating room with limited access, created in this emergency for infected patients. All involved surgeons and OR personnel respected a specific protocol for dressing and undressing as described previously.

\section{Patients needing hospitalization for planning a surgical treatment (Fig. 2)}

This group included patients with a diagnosis of a musculoskeletal lesion that required surgical treatment in the subsequent days. The clinical pathway started with body temperature screening and evaluation of flu-like symptoms. A single-bed recovery room was present in the emergency department, the patient was placed in this room and the medical doctor performed the oropharyngeal swab, using the PPE previously described. The patients were then admitted to a dedicated ward, to wait for the 


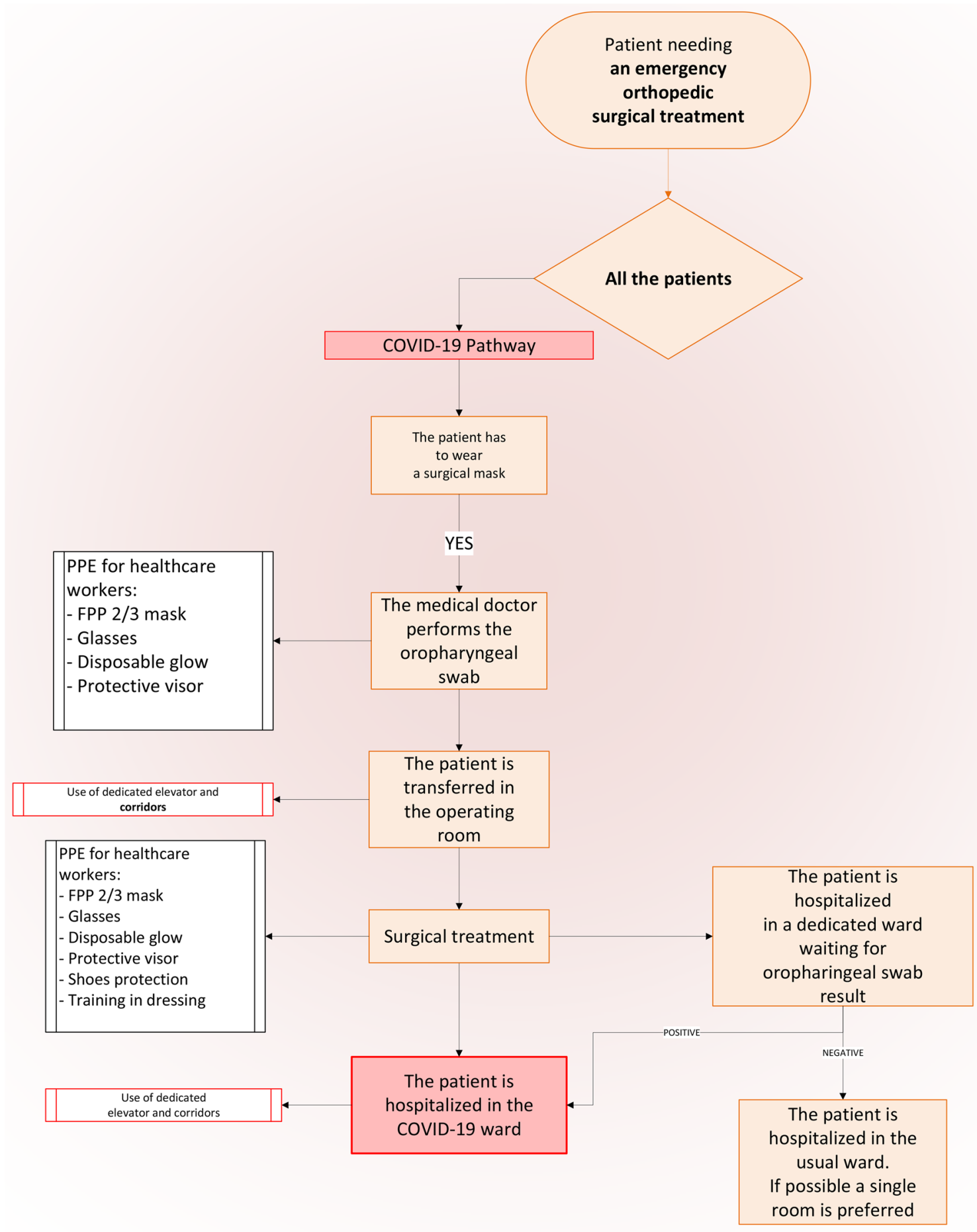

Fig. 1 Flowchart for patients referring to the emergency department and requiring an emergency surgical treatment

oropharyngeal swab result. Patients who had a positive result were admitted to the COVID-19 ward, following the dedicated pathway. Patients with negative tests were then admitted to the usual ward, choosing single rooms when available.

\section{Patients treated conservatively}

This group included a large number of traumatic diseases treated conservatively with plaster, without the need for hospitalization. The clinical pathway started with body temperature control and evaluation of flu-like symptoms. 


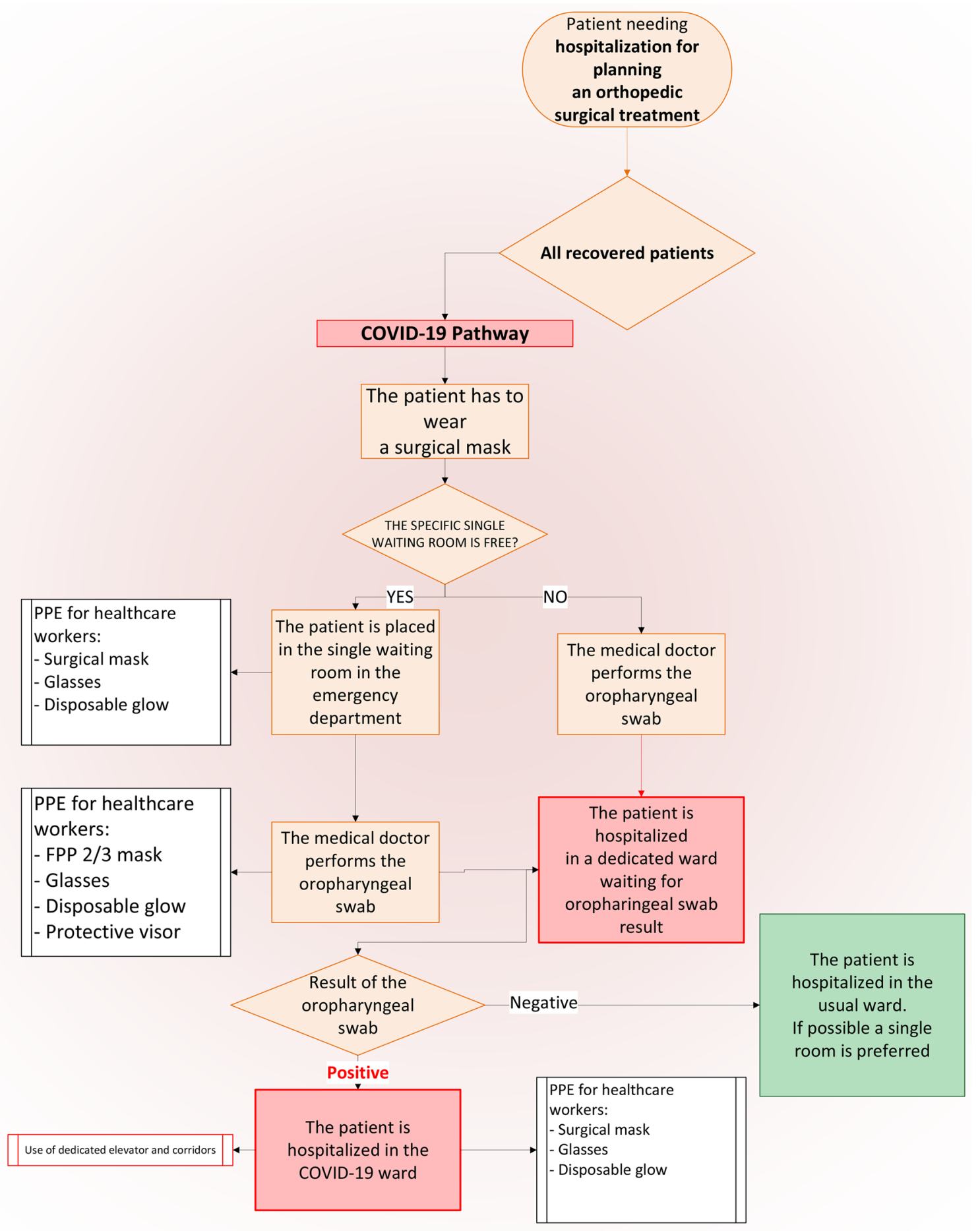

Fig. 2 Flowchart for hospitalization of patients referring to emergency department needing to plan a surgical treatment

Patients without these conditions were treated as usual, discharged home and specific recommendations given for the specific problem, and a visit to the outpatient clinic if necessary. All patients were required to wear a surgical mask during the emergency department stay and all healthcare workers used a surgical mask, glasses and disposable gown when treating this patient. After performing the diagnostic evaluation, the patient was discharged home, given specific recommendations for the specific problem, and a visit to the outpatients department if necessary. The patient were required to contact his general practice medical doctor as soon as possible to monitor the symptoms 
and the hospital office sent a report of the patient's condition to the regional health organization.

\section{Patient discharge}

Patients' discharges had many critical aspects that had to be considered during the COVID-19 pandemic. Hospital internal protocols described the correct flowchart that had to be respected before the discharge of patients recovered from COVID-19 infection or patients that had exposure or contact with patients positive to the virus.

\section{Patients positive to COVID-19}

Discharge criteria for patients positive for COVID-19 were the absence of fever for $72 \mathrm{~h}$, oxygen saturation $>96 \%$ (94\% in case of chronic obstructive pulmonary disease, COPD), respiratory rate $<22$ breaths for a minute and a final negative chest X-ray.

Patients with adequate assistance could be discharged home, formal communication was made with the local health organization, using a specific form describing the clinical situation and the indication for obligatory quarantine.

Patients without a safe home situation were transferred to the military hospital of Milano, converted to a quarantine centre during the pandemic COVID-19.

\section{Patients that had exposure or contact with patients positive to the COVID-19}

Discharge of patients without fever or flu-like symptoms that had close contact with a person positive to COVID-19 followed a dedicated protocol. This group included patients with one of the following features: living in the same house, direct physical contact, direct physical contact with droplet exposure of positive COVID-19, staying in the same room for more than $15 \mathrm{~min}$, a healthcare professional that had contact with positive COVID-19 patient without the protection of correct PPE. This group of patients was discharged home, after formal communication with the local health organization using a specific form describing the clinical situation and indication for obligatory quarantine.

\section{Conclusion}

The Coronaviruses disease (COVID-19) epidemic in the Lombardy region required an immediate re-organization of the local healthcare system. The orthopaedic hub structure proposed during the epidemic could help to improve the quality of assistance for patients with musculoskeletal disorders and reduce the risk of overload in general hospitals. The approach adopted could be used as a starting model for managing the emergency in different healthcare systems, with adaptations due to each regional organization.

Funding No funding was received for this study.

\section{Compliance with ethical standards}

Conflict of interest The authors declare that they have no conflict of interest

Ethical approval This article does not contain any studies with human participants or animals performed by any of the authors.

\section{References}

1. Grasselli G, Pesenti A, Cecconi M (2020) Critical care utilization for the COVID-19 outbreak in Lombardy, Italy: early experience and forecast during an emergency response. JAMA. https://doi. org/10.1001/jama.2020.4031

2. McCloskey B, Zumla A, Ippolito G, Blumberg L, Arbon P, Cicero A, Endericks T, Lim PL, Borodina M, WHO Novel Coronavirus-19 Mass Gatherings Expert Group (2020) Mass gathering events and reducing further global spread of COVID-19: a political and public health dilemma. Lancet. https://doi.org/10.1016/ S0140-6736(20)30681-4

3. Neher RA, Dyrdak R, Druelle V, Hodcroft EB, Albert J (2020) Potential impact of seasonal forcing on a SARS-CoV-2 pandemic. Swiss Med Wkly. https://doi.org/10.4414/smw.2020.20224

4. Singhal T (2020) A review of coronavirus disease-2019 (COVID19). Indian J Pediatr 87(4):281-286

5. Spina S, Marrazzo F, Migliari M, Stucchi R, Sforza A, Fumagalli R (2020) The response of Milan's emergency medical system to the COVID-19 outbreak in Italy. Lancet 395(10227):e49-e50

Publisher's Note Springer Nature remains neutral with regard to jurisdictional claims in published maps and institutional affiliations. 